Rev. Hist., N²7, vol. 1, Enero-Junio 2020: 190-211

ISSN 0717-8832

https://doi.org/10.29393/RH27-9DMC20009

\title{
Desarrollismo y diseño institucional de la Alianza para el Progreso en Argentina
}

\section{Developmentalism and institutional design of the Alliance for Progress in Argentina}

\author{
María Victoria Carsen* \\ Horacio García Bossio*
}

\section{RESUMEN}

En nuestra investigación se sostiene que la promoción del desarrollo fue un elemento compartido de la agenda de Argentina y de Estados Unidos, y entendemos a la Alianza para el Progreso (ALPRO) como un lugar de encuentro, no como un "programa impuesto por los Estados Unidos a la América Latina", sino como un conjunto de ideas latinoamericanas recreadas por los Estados Unidos en una política de cooperación. Proponemos una aproximación al diseño institucional de la Alianza para el Progreso en su aplicación en Argentina, país al cual los organizadores consideraban más que como un mero participante, un actor con "liderazgo y responsabilidad" para promover su avance. Nuestra mirada de las relaciones internacionales nos permite reconocer la existencia de múltiples agentes en la construcción de los vínculos entre los países, por eso nos interesa rescatar y contextualizar la participación de la Argentina como actor local para aproximarnos a conocer el grado de compromiso del país con el programa.

Palabras claves: Alianza para el Progreso- cooperación- desarrollismo- diseño organizacional

\section{ABSTRACT}

In our research it is argued that the promotion of development was a shared element of the agenda of Argentina and the United States and we understand the Alliance for Progress as a meeting place, not as a "program imposed by the United States on America Latin", but as a set of Latin American ideas recreated by the United States in a policy of cooperation. We propose an approach to the institutional design of the Alliance for Progress in its application in Argentina, a country that the organizers considered more than as a mere participant, an actor with "leadership and responsibility" to promote their progress. Our look at international relations allows us to recognize the existence of multiple agents in the construction of ties between countries, so we are interested in rescuing and contextualizing the

\footnotetext{
* Licenciada en Historia (UCA). Profesora Adjunta Historia Contemporánea 1, Pontificia Universidad Católica Argentina. La Alianza para el Progreso en Argentina es el tema de tesis del Doctorado en Historia que la Lic Carsen tiene en curso en la Universidad Católica Argentina, bajo la dirección del Dr. David Sheinin (Trent University) y codirección del Dr. Horacio García Bossio (UCA). Este trabajo fue posible gracias a la Beca de Perfeccionamiento Académico de la Universidad Católica Argentina y al Arthur Schlesinger Jr. Research Fellowship que le permitieron consultar la documentación del Centro de Documentación e Información del Ministerio de Hacienda y Finanzas Públicas de la República Argentina y de la JFK Library de Boston, Estados Unidos. Email: mvcarsen@uca.edu.ar

** Doctor en Ciencias Políticas (Pontificia Universidad Católica de Argentina, Licenciado en Historia (UNLP), director del Departamento de Historia, Facultad de Ciencias Sociales, Pontificia Universidad Católica Argentina. Email: horacio_garciabossio@uca.edu.ar
} 
participation of Argentina as a local actor to get closer to knowing the degree of commitment of the country with the program.

Keywords: Alliance for Progress (ALPRO)- cooperation- developmentalism- organizational design

Recibido: Julio 2019

Aceptado: diciembre 2019

\section{Introducción}

Cuando en unas convulsionadas elecciones de 1958 fue elegido como presidente argentino Arturo Frondizi, este asumió no solo la primera magistratura para gobernar el Estado, sino que justificó su accionar desde una visión del mundo (Weltanschauung) desarrollista. Para ello se propuso destrabar junto a su alter ego y asesor Rogelio Frigerio el clivaje peronismoantiperonismo que había sumido a la sociedad argentina en la década precedente, en pos de una síntesis "superadora" y de "centro", encarnada en el denominado movimiento de integración y desarrollo. ${ }^{1}$

Para Catalina Smulovitz, la solución centrista encarnada en el triunfo electoral de la Unión Cívica Radical Intransigente (UCRI, que fue una escisión del centenario partido radical, provocada precisamente por el acercamiento de Frondizi al exiliado ex presidente con el pacto Perón-Frigerio) se basó en abortar la "solución" de la Revolución Libertadora - el gobierno de facto que realizó el golpe en 1955- desplazando a la Unión Cívica Radical del Pueblo (UCRP, la rama tradicional del radicalismo, a la que los militares contaban como segura ganadora) y relanzando al peronismo a la escena política y con él reavivando el clivaje peronismoantiperonismo. Smulovitz señalaba que el peronismo no solo no debía tener injerencia alguna en elecciones (cabe recordar que Perón mandó a votar a Frondizi frente a los que argumentaban el votoblanquismo) sino que debería ser debilitado como actor político independiente. $O$ sea que eliminando a unos de los polos que animaban el clivaje, desaparecería la expresión de máxima tensión y máxima polarización. ${ }^{2}$

Por su parte, Eugenio Kvaternik refleja la "crisis del centrismo", presentando el siguiente modelo heurístico: a) la distribución de las preferencias electorales adoptaría una forma trimodal, con el peronismo en uno de los polos y el antiperonismo en el otro y entre estos dos polos, un partido denominado "centrista" (con un electorado centrista desperdigado en otros "partidos menores" como la UCRI); b) la elección de 1958 puede interpretarse como un

\footnotetext{
${ }^{1}$ García Bossio, Horacio.2016. ¿Qué nos hace más Nación?. Desafíos del desarrollismo frondicista-frigerista, EDUNLA. 2o Edición, Buenos Aires, EDUNLA. (1ํo edición 2014)

2 Smulovitz, Catalina. 1988., Oposición y gobierno en los años de Frondizi 1 y 2, CEAL, Colección Biblioteca Política Argentina, $\mathrm{N}^{\circ} 213$ y 214.
} 
desplazamiento del centro hacia el polo peronista, así como la de 1962 implica un desplazamiento del centro (Frondizi) hacia el polo antiperonista; c) el punto importante consistiría en que, según Kvaternik, a pesar de existir un electorado centrista, ningún partido ocupa el centro. Parafraseando a Sartori afirma que "las tendencias centristas existen siempre, lo que no siempre existe es un partido de centro"; d) el "núcleo duro" de la argumentación de Kvaternik está focalizado en resaltar, por un lado, que la fragmentación del centro (o crisis del centrismo) se traduce en el fracaso del frondizismo; por otro lado, el autor presenta la paradoja de un peronismo proscrito que engendra, desde esa misma proscripción, la ruptura (y la imposibilidad) de consolidación de un centro, pues fueron inútiles tanto los intentos de desmovilizar políticamente a la clase obrera, como aquellos destinados a lograr su "integración" en lo que Frigerio denominaba el "movimiento nacional". ${ }^{3}$

Ahora bien, el desarrollismo argentino tuvo como inspirador a Rogelio Frigerio, quien desde su formación marxista y su papel de Secretario de Asuntos Económicos y Sociales de la presidencia desplegó una suerte de Weltanschauung desarrollista-frigerista. Los principales argumentos de Frigerio los expuso desde un semanario, la revista Qué sucedió en siete días o a través de sus obras más influyentes: Las condiciones de la victoria y Estatuto del subdesarrollo. Algunos de los rasgos más propiamente frigeristas fueron: ${ }^{4}$

a) El carácter estructural, intrínseco y orgánico del subdesarrollo argentino: el subdesarrollo (y el desarrollo como su contracara) no era producto de ningún fatalismo ni era una condición per se apriorística, sino que era la resultante de un proceso histórico dinámico (y dialéctico) que se fue operando en la era de concentración capitalista, que adquiere, según Frigerio, la forma universal del monopolio, como etapa superior de la acumulación. ${ }^{5}$

b) El análisis objetivo de la realidad, entendida dentro de la lógica de un "marxismo nacional": para Frigerio, las categorías conceptuales emanan del estudio de las condiciones objetivas de producción, pero sin caer en el determinismo mecanicista de las posturas tradicionales de izquierda, quienes no consideran el imprescindible carácter nacional del desarrollo.

c) La consolidación del modelo agro-importador: según Frigerio, el peso de la estructura agroimportadora con la cual la Argentina se insertó en la economía mundial capitalista (aceptando la lógica de la división internacional del trabajo), tuvo un impacto fundamental en las clases dirigentes "desde la derecha a la izquierda; conservadores y radicales hasta los peronistas..." ${ }^{6}$.

\footnotetext{
${ }^{3}$ Kvaternik, Eugenio. 2006., Polarización, perspectivas y casos: a la búsqueda de una tipología; Documento de Trabajo № 1, Año 1, Buenos Aires, Instituto de Ciencias Políticas y Relaciones Internacionales; Universidad Católica Argentina. ${ }^{4}$ Frigerio, Rogelio. 1969. “El carácter de la crisis crónica de la economía argentina y la estrategia para superarla”, en Desarrollo y desarrollismo, Editorial Galerna, Buenos Aires, pp. 116 a 118 y pp. 156 a 164.

${ }^{5}$ Ibíd., p. 117

${ }^{6}$ Ibíd., p.116.
} 
Para Frigerio, esas clases dirigentes se confundieron con la "ilusión del progreso indefinido" de la Argentina.

d) La ignorancia del proceso universal de acumulación capitalista: según este "padre" del desarrollismo nacional, no se comprendió cabalmente la concentración de los capitales en unos pocos centros, sede de los grandes monopolios industriales y de los grupos financieros que dominan el mercado mundial.

e) El (sub) desarrollo no es un dato estático, sino que es una relación: dicha relación se daba entre el desenvolvimiento de las economías primarias y de el de las grandes potencias industriales, originándose -según Frigerio- la pauperización de las primeras y el enriquecimiento de las segundas, por el secular deterioro de los términos del intercambio (DTI) ${ }^{7}$. f) El desarrollo consistiría en la transformación de las estructuras primarias: se deberían crear las bases industriales capaces de proveer al agro de los insumos de capital y de tecnología indispensables para su modernización. Pero debido al DTI y a los problemas de estrangulamiento externo, se debería orientar la economía al desarrollo de un modelo ISI, a partir de la integración productiva entre el agro y la industria. ${ }^{8}$ Frigerio, además, insistía que la base de dicha integración era la explotación al máximo de los recursos naturales del país (energéticos, combustibles sólidos e hidrocarburos; minerales siderúrgicos y no ferrosos; productos químicos y petroquímicos, celulosa) para erigir una industria pesada del acero, la química y las "máquinas- herramientas" 9 .

g) Reafirmación de un proceso de integración económica vertical y horizontal: Frigerio aseguraba la verticalidad en la relación complementaria entre agro e industria y la horizontalidad en cuanto a la difusión geográfica integrada de las industrias que le dieran a la Argentina un crecimiento homogéneo, destruyendo la macrocefalia (y la preeminencia) del litoral portuario. La famosa expresión de Frigerio era que la Argentina era más que el “...semicírculo de $300 \mathrm{~km}$ en torno al puerto de Buenos Aires..."10.

h) Despliegue del "nacionalismo de fines" (y no del "nacionalismo de medios"): sin bien el desarrollismo vendría a completar, según una clásica frase de Frigerio, "el Tercer Plan Quinquenal que Perón no pudo realizar", no era incompatible un modelo ISI complejo con la posibilidad de las IED.

\footnotetext{
7 lbíd., p. 117.

8 Ibíd., p 157.

${ }^{8}$ Ibíd., p. 158

${ }^{10}$ Revista QUE, Año 2, № 99, 4 de septiembre de 1956, p. 3.
} 
i) Planificación económica del Estado desarrollista: para lo cual Frigerio diseñó una usina intelectual (a la manera del Instituto Superior de Estudios Brasileños, ISEB, de Helio Jaguaribe en el Brasil) desprendida de un grupo de estudiosos (los "frigeristas" del CIN, Centro de Investigaciones -más tarde de Estudios- Nacionales, de la avenida Luis María Campos), consolidado a modo intelligenzia dentro de un muy cuestionado entorno presidencial. Un reducto de think tank, pretenciosamente similar a la prestigiosa London School of Economics and Political Science, se conformó con 150 especialistas (muchos de los cuales serían columnistas claves de Qué) entre sociólogos, economistas, estadísticos, juristas y escritores, quienes -según Szusterman ${ }^{11}$ - se organizaban en 30 comités.

La integración se tornaba, pues, en la llave que abriría el ansiado take off de un país que (siguiendo las tesis de Walt W. Rostow) pretendía superar su atraso estructural de una economía primaria exportadora para convertirse en un polo de desarrollo industrial en el Cono Sur. Dicha integración tenía tres aspectos y era la condición sine qua non para el desarrollo. Se hablaba de: integrar a los distintos sectores sociales como una estrategia de superación de la lucha de clases; integrar a las distintas economías regionales en un proyecto integral de desarrollo y, finalmente, la integración presuponía la conformación de un mercado común (al modo de la Comunidad Económica Europea, nacida del Tratado de Roma de 1957) junto a sus vecinos sudamericanos -especialmente el Brasil desarrollista de Kubistchek-; Frondizi también vio en la elección de John F. Kennedy y su propuesta de una Alianza para el Progreso (ALPRO) lo comprensión por parte de Estados Unidos que el mejor camino era la cooperación panamericana para el crecimiento, la democracia y el bienestar.

\section{Diseño institucional de la Alianza para el Progreso (ALPRO) en Argentina}

En este apartado se presenta una aproximación al diseño institucional de la Alianza para el Progreso (ALPRO) cuando se intentó su implementación en Argentina, país al cual los organizadores consideraban más que un mero participante, ya que lo pensaban como un actor con "liderazgo y responsabilidad" para promover su avance ${ }^{12}$. Nuestra mirada de las relaciones internacionales nos permite reconocer la existencia de múltiples agentes en la construcción de los vínculos entre los países ${ }^{13}$, por eso nos interesa rescatar y contextualizar la participación

\footnotetext{
${ }^{11}$ Szusterman, Celia. 1986. Frondizi la política del desconcierto, Buenos Aires, EMECE, p. 142

12 La Argentina y la Alianza para el Progreso. Obras en ejecución, Buenos Aires, s/f.

${ }^{13}$ Kaplan, Amy y. Pease Donald E (comp).1993. Cultures of United States Imperialism, Durham, N.C., Duke University Press, 1993; Pratt, Mary Louise. 1997. Ojos imperiales. Literatura de viaje y transculturación, Bernal, Universidad Nacional de Quilmes, 1997; Gilbert, Joseph; LeGrand, Catherine y Salvatore Ricardo (eds.).1998.: Close Encounters of Empire. Writing the Cultural History of US-Latin American Relations, Durham, Duke University Press; Salvatore, Ricardo.2005. Culturas imperiales. Experiencia y representación en América, Asia y África, Rosario, Beatriz Viterbo Editora, 2005.
} 
de la Argentina como actor local para aproximarnos a conocer el grado de compromiso del país con el programa.

A nivel hemisférico el gran desafío que enfrentó la ALPRO fue encontrar armonía entre los intereses de la política de Estados Unidos y los de los países latinoamericanos, a la vez que se debían considerar las necesidades y expectativas diferenciadas en tan vasto y heterogéneo conjunto. Brasil y Argentina eran vistas como naciones claves por tener entre ambas casi la mitad de la población de la región. ${ }^{14}$

Aunque es frecuente que la ALPRO sea concebida como un programa unilateral de Estados Unidos -tanto sea en su concepción como en su gestión práctica- lo que buscamos proponer aquí es que en primera instancia el programa recogió reclamos que provenían de gobiernos latinoamericanos y en segundo lugar fue adoptado y adaptado por ellos de acuerdo a sus necesidades y singularidades. ${ }^{15}$ Es decir que podemos ver la promoción del desarrollo como un elemento compartido de la agenda de Argentina y de Estados Unidos y a la ALPRO como un lugar de encuentro; no como un "programa impuesto por los Estados Unidos a la América Latina", sino como un conjunto de ideas latinoamericanas recreadas por los Estados Unidos en una política de cooperación. ${ }^{16}$ Los gobiernos radicales de la época se manifestaron en esta sintonía.

Cabe señalar que la experiencia de estos gobiernos tuvo dos caras contrapuestas: primero durante la presidencia del radicalismo intransigente de Frondizi (1958-1962) se celebró la ALPRO como "una nueva perspectiva histórica a la tarea común de las repúblicas americanas" a la vez que un revival de "las mejores tradiciones de una gran nación al tomar una iniciativa proporcionada a la significación y la urgencia del problema" del subdesarrollo. Frondizi se presentó ante Kennedy como "irrevocablemente partícipe de la Alianza para el Progreso" aunque no dejara de señalarle la urgencia de expandir las industrias y tecnificar la agricultura para evitar el riesgo de orientar la cooperación internacional solo a la subsistencia física, es decir, atacar el hambre, las enfermedades contagiosas y el analfabetismo. ${ }^{17}$

Luego del asesinato de Kennedy en 1963, Carlos Perette, el vicepresidente del presidente radical del pueblo Arturo Illia (1962-1966), se refirió así a la relación entre ambos países:

“...han estado y estarán identificados en ideales comunes en defensa del decoro humano y por el imperio de la democracia, de la paz, de la justicia y de la igualdad, de cuyos principios

\footnotetext{
14 AID: U.S. Foreign Aid in the Alliance for Progress, 1965, p. 4.

15 Estas ideas se vieron plasmadas, por ejemplo, en documentos como el informe de Juscelino Kubitschek para la creación de la Operación Panamericana (1958) o el Acta de Bogotá (1960).

16 Theberge, James. 1964. La estructura internacional de la Alianza para el Progreso, Buenos Aires, Fundación de Investigaciones Básicas de la Realidad Americana, Buenos Aires, 1964, p. 7.

17 Carta de Arturo Frondizi a John F. Kennedy, 3 de abril de 1961.
} 
fueron dignos abanderados Washington, Jefferson, Lincoln, Roosevelt, Kennedy, y que aquí en nuestro país sostiene hoy con firmeza el gobierno nacional que preside el doctor Illia"... ${ }^{18}$

\section{La planificación como clave para el diseño institucional}

La planificación fue el instrumento elegido por la ALPRO "para proyectar e implementar un esfuerzo de desarrollo eficaz y consistente" ${ }^{19}$. Esta época fue de apogeo del entusiasmo por la planificación económica en ambos bloques contendientes en la Guerra Fría. ${ }^{20}$ Los documentos oficiales de la ALPRO identificaban la necesidad de establecer en Estados Unidos un organismo unificado para la asistencia externa y fortalecer o crear (en los casos en los que no existieran) instituciones dedicadas a la planificación en los países latinoamericanos. ${ }^{21}$

Si bien ya estaban activos en la región organismos internacionales dedicados a la planificación y a la asistencia externa ${ }^{22}$, en América Latina el concepto mismo de planificación como forma de estimular el cambio social y económico resultaba completamente nuevo. En 1961 solo México, Chile y Ecuador habían dado algunos pasos firmes en este sentido; Argentina se encontraba muy rezagada. ${ }^{23}$

A pesar de que desde 1948 la Comisión Económica para América Latina (CEPAL) venía trabajando en el tema de la planificación económica, ${ }^{24}$ en la región existían falencias técnicas y políticas para afrontar esta tarea. Para respaldar los esfuerzos individuales de cada nación, la CEPAL incluso creó en 1962 el Instituto Interamericano de Planificación Económica y Social con

\footnotetext{
${ }^{18}$ El Senado de la Nación y sus homenajes a Su Santidad el Papa Juan XXIII y al Presidente de los Estados Unidos de América John F. Kennedy, Buenos Aires, Impr. del Congreso de la Nación, 1964.

19 Perloff, Harvey y Sáez, Raul. 1963. "Planificación nacional y multinacional en la Alianza para el Progreso", preparado para la Conferencia de las Naciones Unidas sobre la Aplicación de la Ciencia y la Tecnología en Beneficio de las Naciones menos Desarrolladas., p. 2.

${ }^{20}$ Ardnt, Heinz. 1992. Desarrollo Económico. La historia de una idea, Buenos Aires, Rei Argentina, p. 72.

${ }^{21}$ La planificación y el diseño institucional constituyen un objeto de interés y reflexión para los promotores de la Alianza para el Progreso; entre los documentos oficiales escritos al respecto podemos señalar Planificación Nacional y Multinacional en la Alianza para el Progreso y La organización de instituciones y la Alianza para el Progreso.

$22 \mathrm{Al}$ situarnos en el período posterior a la Segunda Guerra Mundial vemos surgir el concepto mismo de asistencia al desarrollo internacional y encontramos que se habían establecido más de 200 organizaciones públicas y privadas para iniciar programas de cooperación técnica interamericana. Entre las más importantes podemos mencionar: el Banco Mundial, la Corporación Financiera Internacional, la Asociación de Desarrollo Internacional, el Fondo Monetario Internacional, el Fondo Especial para las Naciones Unidas, la Comisión Económica de las Naciones Unidas para América Latina, el Banco Interamericano de Desarrollo, la Organización de los Estados Americanos, la Agencia Internacional para el Desarrollo, el Export-Import Bank, el Programa Alimentos para la Paz, los Cuerpos de Paz. Nystrom, J. Warren y Haverstock, Nathan.1966: The Alliance for Progress, key to Latin America's development, Princeton, N.J., Van Nostrand, p. 57

${ }^{23}$ Accomplishments under the Alliance, Alliance for Progress, 11/1965, Robert F. Kennedy Senate Papers, Box 9, Series Trips, 1964-1966, John F. Kennedy Library (JFKL).

${ }^{24}$ Montuschi, Luisa y Vázquez, Vicente. 1970.Presedo: Plan y laissez-faire, en la economía contemporánea, Buenos Aires, Ediciones Macchi, 1970. p. 93.
} 
sede en Santiago de Chile. Su finalidad era contribuir a la formación de técnicos y especialistas en el área de la programación y asesorar a los gobiernos nacionales a establecer los organismos que estarían a cargo de esta tarea. Esto se hacía con la expectativa de desarrollar en los países de la región un "criterio técnico" para canalizar en forma eficiente la ayuda externa que se recibiría. ${ }^{25}$

Era claro que existía una gran brecha entre los ambiciosos objetivos de la ALPRO y los limitados recursos financieros y técnicos que padecían muchos países latinoamericanos, por ello se creía que con la planificación se podía acortar esa distancia. El diseño institucional entonces tenía como objetivo último lograr que el desarrollo se organizara en cada uno de los países firmantes de la Carta de Punta del Este (agosto de 1961) así como a nivel multinacional, evitando la superposición de proyectos.

El éxito de la asistencia externa para el desarrollo ya se había demostrado con el Plan Marshall (Economic Recovery Plan) que había dirigido \$13 billones de dólares para la reconstrucción de Europa. ${ }^{26}$ Las comparaciones entre la asistencia brindada al viejo continente después de la Segunda Guerra y la que se ensayaba ahora para América Latina se volvieron inevitables entre los contemporáneos, aunque los académicos no lleguen a un consenso sobre el Plan Marshall como modelo para el diseño de la ALPRO. ${ }^{27}$ Por un lado Europa contaba ya con mecanismos financieros, técnicos y políticos sólidos por lo tanto requería solo una asistencia marginal que respaldara el proceso de recuperación económica. ${ }^{28}$ Por otro lado, la naturaleza y cantidad de ayuda económica fue significativamente menor en el caso de la ALPRO. ${ }^{29}$ Un editorial de la revista LIFE de marzo de 1961 señalaba:

"This Marshall Plan analogy can be grossly misleading. In contrast with postwar Europe, where massive dollar aid soon put a damaged but viable system on its feet, most Latin American countries need a lot of things worse than dollars. They need the institutional

\footnotetext{
${ }^{25}$ CEPAL .1962. "El Instituto Latinoamericano de Planificación Económica y Social”, Boletín Económico de América Latina, Volumen VII, no 2, Santiago de Chile., octubre de 1962.

${ }^{26}$ Thomas M. Leonard: 2006. "United States Agency for International Development (USAID)", Encyclopedia of the Developing World, Nueva York, Routledge, p. 1635.

27 Para un detalle sobre los autores que debaten si el Plan Marshall fue modelo para la Alianza o por el contrario fue deliberadamente ignorado, ver Selverston Marc. J. (ed.) .2014, A companion to John F. Kennedy, Wiley Blackwell.

${ }^{28}$ Giglio, James N y Rabe Stephen. 2003: Debating the Kennedy Presidency, Lanham (Maryland), Rowman \$ Littlefield Publishers, 2003, p. 49; Sommerfield Raynard M. 1966.: Tax Reform and the Alliance for Progress, 1966 (Ebook, University of Texas Press).

${ }^{29}$ La mayoría de la ayuda para América Latina llegó como préstamos (70 \%), mientras que en el caso europeo los fondos del Plan Marshall fueron en un 90\% donaciones. Thomas, Leonard, et. al. (eds).2012.): "Alliance for Progress", Encyclopedia of U.S- Latin American Relations, Vol. 1, Thousand Oaks (California), SAGE Publications, 2012, p. 10.
} 
framework of a modern economy: fairer tax laws, fairer land distribution, more literacy, better discan and monetary policies, better housing techniques and sanitation". ${ }^{30}$

De aquella experiencia destinada a Europa sí se tomaba ahora la idea de elaborar planes a nivel nacional como punto de partida para solicitar la asistencia y la dimensión multilateral en la instancia de evaluación y aprobación de los planes de desarrollo a cargo de una comisión internacional. ${ }^{31}$ En el esquema de la ALPRO el procedimiento se iniciaba con la elaboración de un plan nacional por cada país, continuaba con la revisión del mismo por el Comité de los Nueve y se concretaba con la respuesta de las agencias externas de financiamiento. De este modo, se constituyó una red interconectada similar a un trípode:

Esquema 1. El trípode de la Alianza para el Progreso

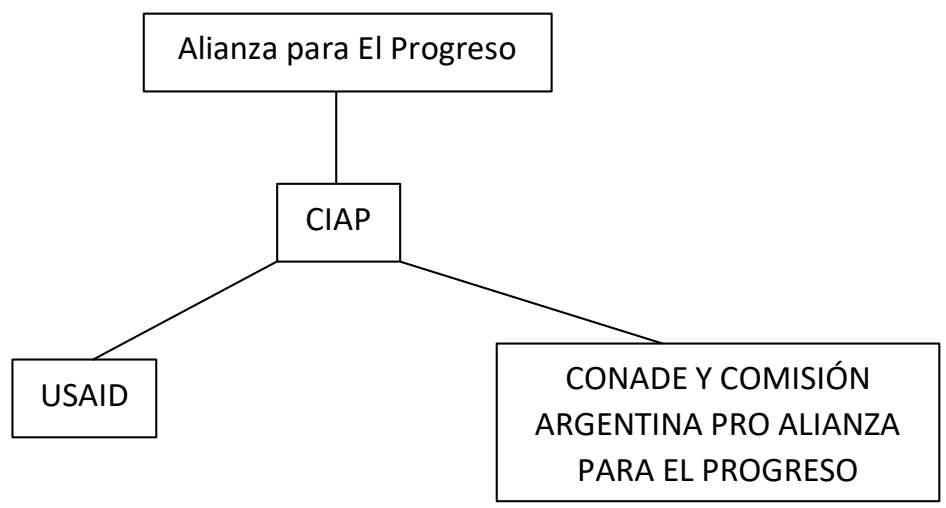

Fuente: elaboración propia partir de "El Plan Kennedy", Editorials, LIFE Magazine, 24 de marzo de 1961, p. 3

El primer pilar de la ALPRO -e institución norteamericana central en su diseño- fue la Agencia Internacional de Desarrollo (USAID) creada por la Ley de Asistencia Externa de 1961. En el marco de la revitalización de los programas de ayuda externa de Estados Unidos se creó este organismo, dependiente del Departamento de Estado, para canalizar la asistencia como forma de diplomacia internacional. Esta agencia estaba activa en todo el mundo y en América Latina canalizó las tareas de la ALPRO. ${ }^{32}$

\footnotetext{
30 "El Plan Kennedy", Editorials, LIFE Magazine, 24 de marzo de 1961, p. 30.

31 Perloff, Harvey y Sáez, Raúl. 1963.: Planificación nacional y multinacional en la Alianza para el Progreso, p. 2.

32 The Story of AID, Washington DC, AID, 1963.
} 
Así se dispusieron por primera vez de mecanismos de planificación que resolvieron los problemas de organización que tenían los programas existentes. Hasta ese entonces Estados Unidos contaba con organizaciones y programas dispersos. ${ }^{33}$ La orden ejecutiva de Kennedy reunió en una sola agencia a los organismos dedicados a la ayuda externa ${ }^{34}$ para promover en forma directa la asistencia técnica y económica de largo alcance, con el foco puesto en la planificación país por país. ${ }^{35}$

La Carta de Punta del Este consideraba los recursos externos como un complemento de las reformas que debían hacerse en cada país a nivel social, económico y político. ${ }^{36}$ Por eso el segundo pilar de la ALPRO fueron, a nuestro entender, las instituciones latinoamericanas. Sus gobiernos debían demostrar voluntad y dar pasos concretos para hacer reformas estructurales que les permitieran maximizar los beneficios de los recursos otorgados.

Para darle un uso eficiente a los recursos se estableció la última pata del trípode, el Comité Interamericano de la Alianza para el Progreso (CIAP) formado en $1963 .{ }^{37}$ Fue el principal órgano multilateral de la Alianza y comité permanente del Consejo Inter-Americano Económico y Social $(\mathrm{CIES})^{38}$. Su función era, por un lado, coordinar la tarea de la comunidad financiera internacional

\footnotetext{
33 En 1950, el programa de los Cuatro Puntos de Harry Truman, se propuso crear mercados para Estados Unidos con lo cual se buscaría reducir la pobreza y aumentar la producción en los países en desarrollo a la vez que se debilitaría el avance del comunismo en estos países. En esta línea la asistencia de Estados Unidos se enfocó en diversos programas de asistencia y se crearon instituciones precursoras de la USAID, como la Mutual Security Agency, Foreign Operations Administration e International Cooperation Administration. USAID, https://www.usaid.gov/who-weare/usaid-history (visitado en julio de 2017).

34" The Foreign Assistance Act of 1961, as amended", Legislation on Foreign Relations Through 2002, http://pdf.usaid.gov/pdf_docs/pcaab142.pdf (visitado en julio de 2017).

${ }^{35} \mathrm{El}$ enfoque de la flamante USAID estaba orientado por la llamada Teoría de la Modernización, difundida, entre otros, por profesores de la Universidad de Harvard y del MIT como Walt Rostow, Max F. Millikan, Lincoln Gordon y John N. Plank. Los supuestos presentados por ella fueron, en primer lugar, formulados en el ámbito de las ciencias sociales, pero luego pasaron a convertirse en ideología y a orientar la vinculación de Estados Unidos con el mundo no-europeo. Ver Walt Rostow y Max Millikan. 1957A Proposal: Key to an effective foreign policy, Nueva York, Harper and Bros; Walt Rostow.1960. The Stages of Economic Growth: A Non-Communist Manifesto, Cambridge, Cambridge University Press; Max F. Millikan, Donald L.M. Blackmer. 1961 The emerging nations: their growth and United States policy, Massachusetts Institute of Technology, Center for International Studies.

${ }^{36}$ Ver "El esfuerzo interno y las necesidades de financiamiento externo para el desarrollo de Argentina", Comité Interamericano de la Alianza para el Progreso, Consejo Interamericano Económico y Social, 1968.

${ }^{37} \mathrm{Y}$ en este mismo sentido se señalaban los proyectos en torno al Mercado Común Centroamericano (CACM) conformado por El Salvador, Guatemala, Honduras, Nicaragua y Costa Rica y la Asociación Latinoamericana para el Libre Comercio (LAFTA, en sus siglas en inglés).

38 El CIES estaba subordinado a la OEA y su tarea era promover desde 1948 el bienestar económico y social americano, había sido reorganizado en 1961 para alcanzar los objetivos de la Alianza. Ver Enrique Domenech. 1971 The Alliance for Progress and the Inter-american Committee of the Alliance for Progress, Washington,
} 
con la de los países y por otro coordinar a la OEA -que mostró escaso entusiasmo ${ }^{39}$ - con el Banco Interamericano de Desarrollo (BID) y la CEPAL.

\section{USAID y su misión en Argentina}

Las misiones de la USAID en cada país eran las encargadas de administrar los préstamos y la asistencia técnica en estrecha relación con los organismos técnicos del gobierno nacional, que en el caso argentino fue el Consejo Nacional de Desarrollo (CONADE), sobre el que volveremos más adelante. Resulta evidente la influencia de esta asistencia externa en la vida política del país. La USAID se atribuía, por ejemplo, el haber creado las condiciones necesarias para que se realizaran las elecciones presidenciales del mes de julio de 1963 que llevaron a Arturo Illia a la presidencia, ya que explicaban la normalización de la vida democrática argentina por el impacto positivo de la asistencia de emergencia recibida. ${ }^{40}$

Luego de la caída de Frondizi por el golpe de Estado de 1962, el Departamento de Estado de Estados Unidos veía que las dos principales amenazas a las instituciones argentinas venían de parte de los militares y de los peronistas. Estos sectores eran caracterizados como extremistas y capaces de eclipsar el esfuerzo de los "reformadores". Un informe de 1962 señalaba:

"In spite of our limited ability to influence the course of political events in Argentina, it is in the interests of the US to encourage by whatever means the forces of moderation within these two groups, and to demonstrate our confidence in the civilian sector of the government. It would not seem wise at the present time to encourage political solidarity within either the armed forces (through any sort of "modernization" program) or the labor movement (by strengthening the authority of the CGT)". ${ }^{41}$

La ayuda extranjera, desde esta perspectiva, venía a financiar programas de política social que se habían abandonado luego de la caída del peronismo y de alguna manera funcionaba como soporte de un proyecto político alternativo. La USAID estableció asuntos claves de acción general que se correspondían con las "áreas de énfasis" señaladas por la Carta de Punta del Este (construcción de viviendas a bajo precio, eliminación del analfabetismo, expansión de la educación en todos los niveles, aumento de acceso al agua potable, reforma agraria, mejora de la productividad, etc). Estos eran los principales puntos de acción: a) producción y exportación agrícola; b) vivienda; c) medios de transporte y comunicaciones; d) desarrollo y administración pública.

\footnotetext{
${ }^{39}$ Nystrom, J. Warren y Haverstock. Nathan A. 1966, The Alliance for Progress, key to Latin America's development, Princeton, N.J., Van Nostrand, p. 61.

40 AID: U.S. Foreign Aid in the Alliance for Progress, 1965, p. 7.

${ }^{41}$ Argentina and the Alliance for Progress, by Roger Hilsman. 5/62-9/62 \& undated, Department of State. Personal Papers of Arthur Schlesinger Jr. White House Files, Box WH 25 Argentina, John F. Kennedy Library (JFKL).
} 
En Argentina la USAID le otorgaba prioridad al primero de estos temas. Los sucesivos directores de la misión eran especialistas en el área de la agricultura. ${ }^{42}$ La primera misión fue encabezada por Albion Patterson, lingüista y docente de profesión, que había sido asesor para el programa de desarrollo agrícola en Chile con la Administración de Cooperación Internacional. A partir de 1964 el director de la USAID en Argentina fue John Heilman, quién tenía antecedentes como funcionario en el Departamento de Agricultura en Washington y en la Asociación Internacional Americana para el desarrollo social y económico de Nelson Rockefeller en Venezuela. A partir de 1968 Richard Lippincott, también con experiencia en el Departamento de Agricultura de Estados Unidos, se desempeñó como representante de la USAID a la cabeza de la lista de funcionarios de la misión en el país.

La exportación de productos agrícolas seguía constituyendo la fuente principal de divisas e incluso el desarrollo industrial estaba de alguna manera condicionado al impulso que se le diera desde el sector agrícola. Por este motivo se ponía el énfasis en la reducción del costo de maquinarias, en las condiciones impositivas que alentaran la inversión de capital en la explotación agrícola y en la obtención de créditos para el sector agropecuario.

En los primeros años de su existencia la misión de la USAID en Argentina se dedicó solo a la asistencia técnica con énfasis en la mejora del ganado y la educación superior. Fue recién hacia mediados de la década de 1960 que fue ampliando sus acciones en el país. En términos generales el aporte de la USAID se vería reflejado en los siguientes aportes: desarrollo de la instrucción secundaria y superior en el campo de la agronomía; extensión de las obras de riego; mejora del servicio gubernamental de extensión agrícola; recursos para el programa argentino de vialidad nacional; construcción directa de viviendas y estímulo a sectores públicos y privados argentinos para colaborar con este fin.

\subsection{La planificación argentina: Consejo Nacional de Desarrollo y Comisión Argentina pro- Alianza para el progreso}

En la Carta de Punta del Este se estableció un período de 18 meses para que cada gobierno preparara un programa nacional a 10 años como estrategia general de desarrollo. Se creía así garantizar que la asistencia extranjera no quedara comprometida a proyectos aislados sino a un plan general compuesto de programas domésticos que movilizarían recursos públicos y privados.

Diseñar este programa implicaba hacer un diagnóstico de la situación económica y social del país para luego establecer áreas prioritarias de acción. Como hemos señalado, desde el planteo inicial de la Carta, se entendía que la planificación podía salvar la gran distancia que existía entre

\footnotetext{
42 Foreign Service List, Departamento de Estado, 1961, 1965 June y 1968; “Obituaries. John Heilman. USAID Official”,
} Washington Post, 27 de abril de 2002, "Obituaries. Richard E. Lippincott", Washington Post, 7 de agosto de 2003. 
los objetivos de la ALPRO y los limitados recursos con los que contaban la mayoría de los países. Un informe indicaba las dificultades encontradas: "In most countries, national development policy making and development investment is not closely tied to, or does not generally emerge from an overall national development plan". ${ }^{43}$

${ }^{43}$ Accomplishments under the Alliance, Alliance for Progress, 11/1965, Robert F. Kennedy Senate Papers, Box 9, Series Trips, 1964-1966, John F. Kennedy Library (JFKL). 
Esquema 2: Diseño organizacional de ALPRO en Argentina

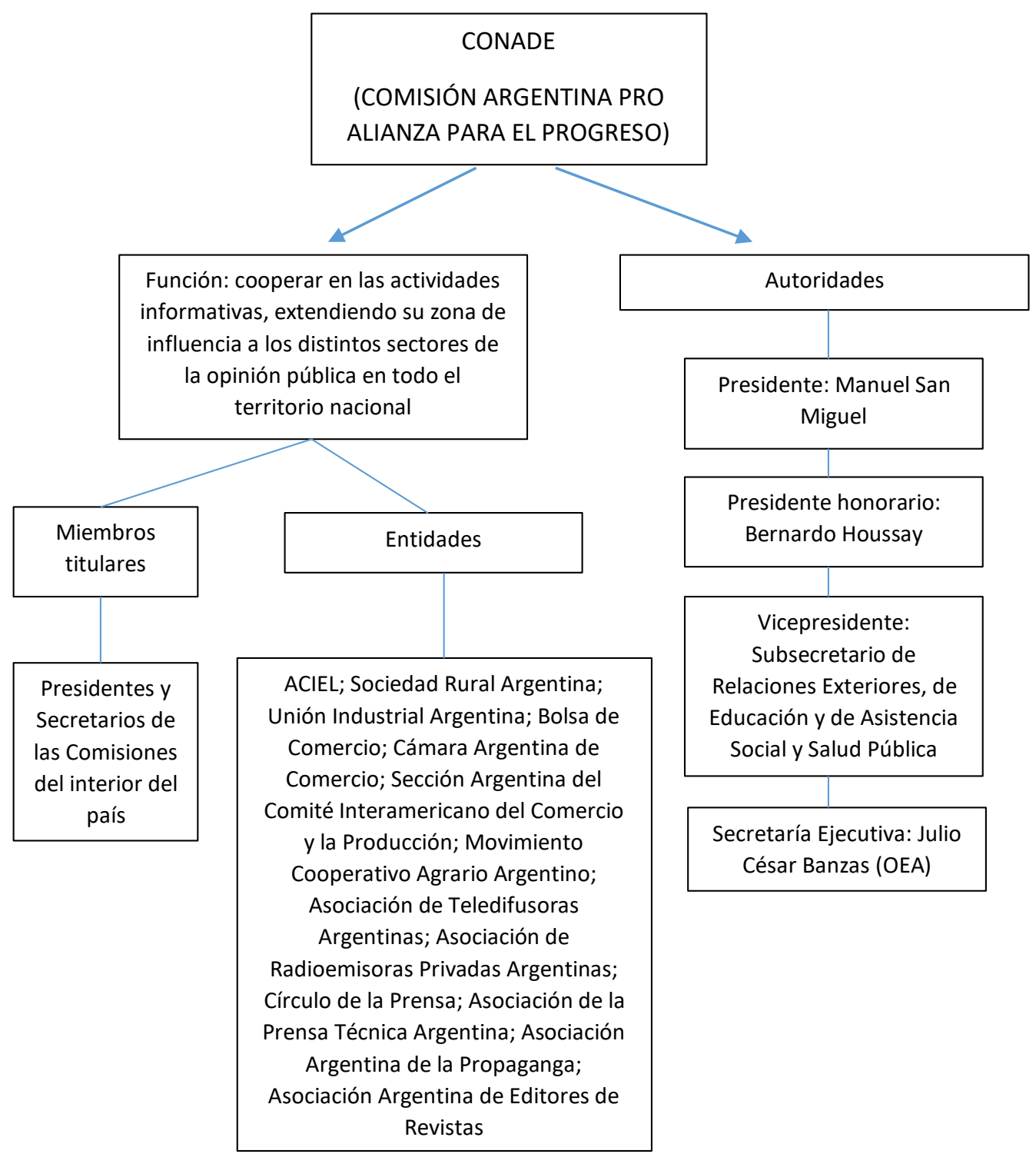

Fuente: elaboración propia a partir de Accomplishments under the Alliance, Alliance for Progress, 11/1965, Robert F. Kennedy Senate Papers, Box 9, Series Trips, 1964-1966, John F. Kennedy Library (JFKL). 
El motor de la planificación lo constituían instituciones de fomento al crecimiento económico. ${ }^{44}$ En algunos países esas instituciones no existían y en otros el obstáculo más pronunciado era la falta de experiencia de personal administrativo para el manejo de las mismas. Visto el vacío generalizado de organismos que pudieran llevar adelante la planificación, la ALPRO estimuló la creación de instituciones para la programación de planes de desarrollo. Hacia 1964 todos los países de la región contaban con organismos de planificación aunque éstos se movían a distinto ritmo. ${ }^{45}$

Si bien más del $75 \%$ de los países estaban ya trabajando en la preparación de planes nacionales, solo seis presentaron sus planes en el tiempo previsto en la Carta. El plan presentado por Venezuela fue el único que cumplía con los elementos requeridos en la Carta de Punta del Este en cuanto a formulación y coordinación de políticas para movilizar los recursos requeridos. El resto habían sido elaborados mayormente por técnicos y carecían de relevancia política ya que no ajustaban los objetivos a las posibilidades reales de realización. Más alarmante aún, carecían en forma casi universal de planes o propuestas para proyectos específicos. ${ }^{46}$ Este panorama demuestra que el trabajo se había iniciado lentamente y con contratiempos. A pesar de los resultados poco satisfactorios, según las autoridades norteamericanas, en la mayoría de los países se había adoptado firmemente la idea de que para lograr el desarrollo era esencial realizar un inventario sistemático de problemas, objetivos, recursos y prioridades. Esto demostraba el compromiso de los latinoamericanos aunque quizás nunca lograran la planificación como estaba planteada en la Carta. ${ }^{47}$

Ahora veamos ¿ qué ocurría en Argentina? De acuerdo al informe presentado al Consejo Interamericano Económico Social (CIES) en 1963 por el gobierno argentino, el grado de preparación para la planificación variaba de acuerdo al área de trabajo, es decir, que el país no contaba con una preparación ni recursos parejos para afrontar el desafío de la planificación. Para el Plan de Viviendas, por ejemplo, contaba en líneas generales con un buen plantel de técnicos. En esta área la asistencia de técnicos extranjeros solo sería necesaria para "puntos muy específicos u orientaciones generales", pero a título de excepción. No se veía la misma fortaleza en la información estadística con la que contaban los técnicos argentinos, que era escasa y dispersa. Se lamentaba que el país no tuviera todavía con un Departamento de

\footnotetext{
44 Perloff, Harvey y Sáez, Raúl .1963.: Planificación nacional y multinacional en la Alianza para el Progreso, p. 1.

45 "Informe sobre el primer año de la Alianza para el Progreso", Jornadas de la Alianza para el Progreso de Tucumán, San Miguel de Tucumán, 30 de marzo al 2 de abril de 1964.

${ }^{46}$ Accomplishments under the Alliance, Alliance for Progress, 11/1965, Robert F. Kennedy Senate Papers, Box 9, Series Trips, 1964-1966, John F. Kennedy Library (JFKL).

47 Accomplishments under the Alliance, Alliance for Progress, 11/1965, Robert F. Kennedy Senate Papers, Box 9, Series Trips, 1964-1966, John F. Kennedy Library (JFKL).
} 
Vivienda que centralizara y ordenara toda la información..$^{48}$ En el área de salud la situación era más precaria. Se veía que la debilidad mayor estaba en la falta de información básica adecuada para los fines de la planificación aunque también se señalaba la falta de personal, en cantidad y en preparación técnica, para llevar adelante esta tarea.

El proceso de elaboración del Plan Nacional no fue ágil y se materializó recién en el año 1964 (Plan CONADE 1965-1969)..$^{49}$ La Argentina tenía su tercer presidente desde el lanzamiento de la ALPRO tres años antes. Pero los pasos dados en 1961 por el gobierno de Frondizi con la creación del Consejo Nacional de Desarrollo (CONADE) fueron el motor necesario para poner en funcionamiento el trabajo. ${ }^{50}$

La acción de este organismo en el impulso al desarrollo nacional estuvo orientada en función de las relaciones económicas con los Estados Unidos y enfocada a canalizar la ayuda gubernamental norteamericana ${ }^{51}$. En el decreto de su creación se establecía que el CONADE dependería directamente de la Presidencia de la Nación y se encargaría de coordinar los estudios necesarios para la elaboración de los programas de desarrollo y brindar asesoramiento para la acción de gobierno. ${ }^{52}$

Su estructura orgánica establecía como presidente del Consejo al Ministro de Economía, compuesto además por un vicepresidente, un secretario ejecutivo y siete consejeros. Podemos resumir las principales funciones del CONADE en estos items: formular las metas de desarrollo del país a corto, mediano y largo plazo; preparar programas anuales de inversión en los sectores básicos de la economía así como proyectos específicos de carácter regional o sectorial; formular y promover los programas nacionales de asistencia técnica para el desarrollo y coordinar su ejecución.

Bajo la dirección de Roque Guillermo Carranza, destacado militante del partido de gobierno especializado en temas de planeamiento ${ }^{53}$ y del economista Bernardo Grinspun como Secretario Ejecutivo y encargado de la dirección concreta del organismo, se organizó un grupo

\footnotetext{
48 Informe de la República Argentina para la Segunda Sesión de la Comisión Especial VI del Consejo Interamericano Económico Social (CIES), Buenos Aires, 1963.

${ }^{49}$ Consejo Nacional de Desarrollo (CONADE): Plan Nacional de Desarrollo 1965- 1969, Buenos Aires, 1965.

50Jáuregui, Aníbal. 2014. “La planificación en la Argentina del desarrollo (1955-1973)", Temas de historia argentina y americana № 22, p. 29.

${ }^{51}$ Núñez, Paula Gabriela y LópezNúñez, Silvana. 2014. López: “Lo rural y lo científico: La presencia de la Estación Experimental Regional Agropecuaria (EERA) INTA Bariloche 1962-1968", Estudios Rurales N 7, pp. 105-126Segundo semestre 2014.

52 El CONADE fue creado por el Decreto No 7.290/61. Un decreto de mediados de 1962 (Decreto no 6337) amplía las atribuciones del CONADE y pone a su cargo el estudio y asesoramiento respecto del plan general de obras, trabajos públicos e inversiones patrimoniales. Sus funciones se amplían nuevamente en 1964 (Decretos no 565 y 566). En 1966 fue nuevamente reestructurado por la Ley 16964 y reducido en sus funciones.

${ }^{53}$ Enrique Pereira, Enrique. 2008. “Carranza, Ing. Roque Guillermo”, Diccionario Biográfico Nacional de la Unión Cívica Radical. Consultado en http://diccionarioradical.blogspot.com.ar/ , julio de 2017.
} 
de alrededor de 200 jóvenes técnicos, especialmente economistas, matemáticos e ingenieros, que recibían el apoyo de Richard Mallon (del Harvard Advisory Group) y Alberto Fracchia (perteneciente a CEPAL). ${ }^{54}$ Para cumplir con las metas trazadas en su diseño original el CONADE trabajó en conjunto con el Consejo Federal de Inversiones (CFI) que se ocupaba de tareas de investigación, coordinación y asesoramiento, el Banco Central de la República Argentina, órgano básico de asuntos financieros y crediticios en el país y el Gabinete Económico Social que tenía como función armonizar "en el más alto nivel gubernamental las decisiones de la política del gobierno que surgen de los Ministerios y organismos estatales claves para el desarrollo económico". ${ }^{55}$

Es así que podemos ver que el impulso inicial de la planificación del desarrollo en Argentina vino del gobierno de Frondizi, durante el cual se creó el organismo y se inició con la elaboración de informes parciales en el área de planificación. El avance fue posible gracias a la incorporación de novedades metodológicas y a la cooperación con otras instituciones y el trabajo conjunto de funcionarios argentinos con extranjeros y la planificación del desarrollo dio sus frutos luego de la asunción a la presidencia de Illía. Así lo señalaba la USAID:

"Development planning has been given increased emphasis through the strenghtening of the National Development Council and contracts with Harvard University and Stanford Reseach Institute. It's expected that with this assistance a national development plan will be completed by the end of $1964 .{ }^{\prime \prime 6}$

Entonces, a fines de 1965 fue presentada la versión revisada del primer Plan Nacional de Desarrollo, que sería considerado por algunos "el intento más serio y sistemático realizado en materia de planificación en el país". ${ }^{57}$ Como lo han expresado otros trabajos, en este Plan se manifiesta la corriente de pensamiento económico que podemos clasificar dentro del paradigma de planificación desarrollista gradualista cuya legitimidad política estaba dada por el "carácter internacional" de la planificación. ${ }^{58}$

\footnotetext{
${ }^{54}$ De Pablo, Juan Carlos. de Pablo.1979., La economía que yo hice, Buenos Aires, Ediciones El Cronista Comercial.

55 "El Consejo Nacional de Desarrollo", Jornadas de la Alianza para el Progreso de Tucumán, San Miguel de Tucumán, 30 de marzo al 2 de abril de 1964.

${ }^{56}$ AID: U.S. Foreign Aid in the Alliance for Progress, 1965, p. 8.

57 Montuschi Vázquez. 1970. P, Plan y laissez-faire, en la economía contemporánea, Buenos Aires, Ediciones Macchi. 58 Tearoti, Silvia Aurora. 2013. Tearoti: El Plan de Desarrollo 1965-1969, un intento de planificación en la Argentina". XIV Jornadas Interescuelas/Departamentos de Historia. Departamento de Historia de la Facultad de Filosofía y Letras. Universidad Nacional de Cuyo, Mendoza., 2013. Martín FISZBEIN, Martín. "Instituciones e ideas en desarrollo. La planificación económica en la Argentina, 1945- 1975" en”, ROUGIER, Marcelo (comp.). 2011. Estudios sobre la industria argentina, Buenos Aires, Lenguaje Claro. Stawski, Martin y Taroncher, Miguel Ángel. "La planificación y el CONADE: un análisis del Plan Nacional de Desarrollo, 1965-1969" en Juan Odisio y Marcelo Rougier (comps.) (2019.), Estudios sobre planificación y desarrollo económico. Aportes para un diseño institucional estratégico, Lenguaje claro Editora, Buenos Aires.
} 
La USAID elogiaba otras iniciativas tomadas por el gobierno de Illia que reflejaban, a su juicio, que el presidente radical estaba en la misma línea de la ALPRO. ${ }^{59}$ La primera medida que se celebraba era la adopción de políticas que mejorarían la producción agrícola, entre ellas la penalización del uso de la tierra no productiva y la incorporación de incentivos impositivos para favorecer el uso más intensivo de la misma. Por otro lado el informe de 1965 de la USAID veía que la reorganización del sistema impositivo argentino podía darle aire al gobierno para reducir la evasión impositiva, favoreciendo la modernización del sistema de recolección de impuestos y comenzando a utilizar equipos de procesamiento de información. ${ }^{60}$

Ciertamente no le es posible al historiador medir los logros que este plan hubiera podido alcanzar dado que su aprobación estaba pendiente hacia mediados de 1966 cuando Illía fue derrocado por la Revolución Argentina (1966-1973). Las nuevas autoridades del país removieron a las del CONADE y anularon la implementación del Plan Nacional de Desarrollo. La fragilidad de la vida política nacional acotó rápidamente las perspectivas de la planificación a largo plazo e hizo que las actividades del CONADE se fueran reduciendo cada vez más al asesoramiento en materia de inversiones a corto plazo. Fue nombrado un interventor militar y la trama burocrática del organismo se hizo sumamente compleja. Las prioridades cambiaron, con un fuerte énfasis en el diseño de objetivos que combinaran la meta del desarrollo con la de la seguridad. ${ }^{61}$ Se elaboraron planes que sucesivamente fueron reemplazando a otros como producto de la misma inestabilidad política que en este caso afectó al gobierno de facto. ${ }^{62}$

Estrechamente vinculado al quehacer del CONADE estuvo la Comisión Argentina Pro-Alianza para el Progreso formada en octubre de 1962, a pedido de la OEA, que impulsaba la formación de comisiones a nivel nacional en los países firmantes de la Carta de Punta del Este. ${ }^{63}$ La Comisión fue un organismo diseñado con criterio federal; entre sus miembros titulares estaban los representantes de comisiones del interior del país. ${ }^{64}$ La presidencia honoraria correspondía al renombrado científico Bernardo Houssay, presidente del CONICET y Premio Nobel argentino. Para favorecer la participación amplia de distintos sectores del gobierno en la Comisión estaban representadas las áreas de relaciones exteriores, educación, asistencia social y salud pública, ya que los vicepresidentes de la Comisión eran los subsecretarios de dichas carteras.

\footnotetext{
${ }^{59}$ AID: U.S. Foreign Aid in the Alliance for Progress, 1965, p. 8.

${ }^{60}$ AID: U.S. Foreign Aid in the Alliance for Progress, 1965, p. 9.

61 El ahora conocido como Sistema Nacional de Planeamiento y Acción para el Desarrollo, coordinado por la Secretaría del CONADE, fue complementado con el Sistema de Planeamiento y Acción para la Seguridad (creado por Ley 16970 y coordinado por el Consejo Nacional de Seguridad.

62 Plan Nacional de Desarrollo (1970-1974) que no se aplica por la caída de Onganía, Plan tentativo nacional de Desarrollo para el período 1971-1975 y Plan Nacional de Desarrollo y Seguridad (1971-1975).

63 La Secretaría Ejecutiva de la Comisión estaba a cargo de Julio César Banzas, Director de la Oficina de Información de la Secretaría General de la OEA en la Argentina.

${ }^{64}$ Hasta 1964 se habían presentado las de las provincias de Santa Fe y Córdoba.
} 
Su presidente, Manuel San Miguel, se desempeñaba como vicepresidente del CONADE y a la vez coordinador del gobierno argentino para todos los programas de la ALPRO. ${ }^{65}$ Doctor en Ciencias Económicas con especialidad en Economía Política, San Miguel se había dedicado a la docencia como profesor de Economía en la Universidad de Buenos Aires (1957-1960) a la vez que había ocupado cargos de importancia a nivel nacional e internacional como la vicepresidencia del Instituto Nacional de Granos y Elevadores y la presidencia de la Delegación Argentina ante el Comité de Comercio de la CEPAL. Además, había ocupado la subsecretaría del Ministerio de Comercio e Industria en los años postperonistas. La Comisión estaba dedicada a cooperar en las actividades informativas de la ALPRO, extendiendo su influencia a los distintos sectores de la opinión pública en todo el territorio nacional. Para asistirla en esta tarea, una serie de instituciones centrales en el quehacer económico de la vida nacional eran miembros de la Comisión, entre los que podemos contar a la Sociedad Rural Argentina, a la Unión Industrial Argentina, a la Bolsa de Comercio y a la Cámara Argentina de Comercio. ${ }^{66}$

Una de las tareas más visibles de la Comisión fue la organización de espacios de discusión sobre la ALPRO, bajo las directivas de la OEA. ${ }^{67}$ El resultado fue la realización de encuentros en la Ciudad de Buenos Aires y en San Miguel de Tucumán para reunir a "voceros y exponentes de las fuerzas vivas de los países, entre ellos, particularmente a representantes autorizados de los gremios y sindicatos, a los líderes de la opinión pública y del pensamiento político y social. ${ }^{68}$

Más importante aún, la Comisión Nacional debía dedicarse al estudio y debate del Plan de Desarrollo de cada país, de acuerdo a los siguientes ejes temáticos: la salud pública, la vivienda, la reforma agraria, la educación, la ciencia y la cultura, el sector privado y la Alianza para el Progreso. Lo que nos lleva nuevamente al tópico de la planificación del desarrollo, que en el ámbito nacional encontraba resistencias en determinados sectores de la opinión pública.

Fue San Miguel quién denunció esta actitud en una conferencia ante la Escuela Superior de Técnica del Ejército brindada en octubre de $1963 .{ }^{69}$ La principal oposición a la planificación, constataba el presidente de la Comisión Argentina, era la identificación de este tipo de

\footnotetext{
${ }^{65}$ Aa.V.v. 1969.Quién es Quién en la Argentina, Buenos Aires, 9 ed, Guillermo Kraft, p. 665.

66 La lista completa de entidades incluye también a ACIEL, a la sección argentina del Comité Interamericano del Comercio y la Producción, al Movimiento Cooperativo Agrario Argentino, a la Asociación de Teledifusoras Argentinas, a la Asociación Radioemisoras Privadas Argentinas, al Círculo de la Prensa, a la Asociación de la Prensa Técnica Argentina, a la Asociación Argentina de la Propaganda y a la Asociación Argentina de Editores de Revistas.

${ }^{67}$ A tales efectos la dirección de la Subsecretaría para Asuntos Culturales, Científicos y de la Información de la Unión Panamericana se convirtió en el cuerpo preparatorio destinado a cooperar con las comisiones nacionales en la organización de las jornadas, enviando información y documentos complementarios y de referencia a la agenda propia y generando luego recomendaciones al respecto.

68 Informe de la República Argentina para la Segunda Sesión de la Comisión Especial VI del Consejo Interamericano Económico Social (CIES), Buenos Aires, 1963.

69 "La preparación y el futuro del plan de desarrollo argentino", conferencia pronunciada por Miguel San Miguel en la Escuela Superior Técnica del Ejército, en octubre de 1963, Jornadas de la Alianza para el Progreso de Tucumán, San Miguel de Tucumán, del 30 de marzo al 2 de abril de 1964.
} 
programaciones económicas con los países llamados "colectivistas". La referencia a las diversas experiencias posteriores a la Segunda Guerra Mundial, sin embargo, le permitió a San Miguel mostrar la planificación no como una tarea propia del comunismo sino como una herramienta técnica que podía proyectar en forma concreta metas y objetivos. Es decir, un "método neutral" para resolver el problema central de la economía argentina, que era la escasez generada por el uso inadecuado de los recursos.

\section{Algunas consideraciones finales}

Como hemos visto en el diseño de la ALPRO la planificación era el punto de partida para la promoción de las políticas de desarrollo y el acceso a la asistencia técnica y económica. El diseño institucional de la Alianza estaba pensado, por lo tanto, para facilitar las tareas de diagnóstico y programación de los planes nacionales que se llevaron adelante en los países latinoamericanos con la asistencia de organismos y técnicos extranjeros.

La aproximación al diseño institucional de la ALPRO que hemos realizado en esta presentación nos ha permitido observar que los gobiernos de Arturo Frondizi y Arturo Illia no solo dieron su apoyo a este programa en el discurso público, sino que dieron pasos concretos en el orden de la gestión y administración para poder cumplir con el objetivo de la planificación nacional. Es así que vemos en la puesta en marcha del CONADE y el Plan Nacional presentado hacia mediados de la década de 1960 la voluntad gubernamental de que la Argentina marchara hacia el desarrollo. Pero también es cierto que las tareas técnicas de ese Consejo tenían que combinarse con la adhesión de diversos sectores sociales, y es allí que vemos la relevancia de la Comisión Argentina Pro-Alianza para el Progreso.

EI CONADE como entidad de naturaleza exclusivamente gubernamental tenía características principalmente técnicas y no ejecutivas, de allí que la composición de la Comisión buscaba ser complementaria a las tareas del CONADE e intentaba reflejar el compromiso que se buscaba en otros sectores de la sociedad: el productivo, el cultural, el educativo, el científico. Y que en conjunto conformaron una de las tres patas del trípode institucional que utilizamos como metáfora en este trabajo para el caso argentino.

En síntesis, la participación real del país con la cooperación interamericana no se puede medir solo por la firma de la Carta de Punta del Este, sino por haber generado las condiciones institucionales propicias para sostener un compromiso activo con el desarrollo y con la Alianza para el Progreso... la Argentina lo hizo, aunque las debilidades institucionales de una democracia débil y tutelada por las Fuerza Armadas le hayan impedido dar ese ansiado salto al desarrollo que lo pudo haber convertido en un faro de prosperidad en Sudamérica. Destino fatal de un país llamado a ser lo que nunca pudo lograr. 


\section{Fuentes y Bibliografía}

\section{Fuentes}

Actas de las Jornadas de la Alianza para el Progreso en Tucuman, San Miguel de Tucumán, del 30 de marzo al 2 de abril de 1964.

Actas de las Jornadas de la Alianza para el Progreso de Argentina. El Plan Nacional de Desarrollo, Buenos Aires, 12 a 14 de agosto de 1963, Comisión Argentina Pro-Alianza para el Progreso.

Agencia Internacional de Desarrollo (AID). 1963. The Alliance for Progress...an American partnership, Agency for International Development, Department of State, Washington D.C., s/f. Agencia Internacional de Desarrollo (AID). 1963. The Story of AID, Washington DC.

Agencia Internacional de Desarrollo (AID). 1965. U.S. Foreign Aid in the Alliance for Progress, Washington DC.

CEPAL.1962. Boletín Económico de América Latina, Volumen VII, no 2, Santiago de Chile, octubre.

Argentina and the Alliance for Progress, by Roger Hilsman. 5/62-9/62 \& undated, Department of State. Personal Papers of Arthur Schlesinger Jr. White House Files, Box WH 25 Argentina, John F. Kennedy Library (JFKL).

Comité Interamericano de la Alianza para el Progreso (CIAP). 1968. "El esfuerzo interno y las necesidades de financiamiento externo para el desarrollo de Argentina", en Informe del Comité de los Nueve, Alianza para el Progreso, Consejo Interamericano Económico y Social.

Consejo Nacional de Desarrollo (CONADE). 1964. Informe sobre el plan nacional de desarrollo 1965 -1969, Buenos Aires.

Consejo Nacional de Desarrollo (CONADE). 1965. Plan Nacional de Desarrollo 1965-1969, Buenos Aires. Consejo Nacional de Desarrollo (CONADE). 1968. Inventario de los proyectos de asistencia técnica correspondientes a la Agencia para el Desarrollo Internacional, Programa de las Naciones Unidas para el Desarrollo y la Organización de Estados Americanos.

Senado de la Nación. 1964. El Senado de la Nación y sus homenajes a Su Santidad el Papa Juan XXIII y al Presidente de los Estados Unidos de América John F. Kennedy, Buenos Aires, Impr. del Congreso de la Nación.

Departamento de Estado (EEUU). 1961, Junio 1965 y 1968. Foreign Service List.

Harvey S. Perloff y Raúl Saez. 1963. "Planificación nacional y multinacional en la Alianza para el Progreso", en Conferencia de las Naciones Unidas sobre la Aplicación de la Ciencia y la Tecnología en Beneficio de las Naciones menos Desarrolladas, conferencia celebrada en Ginebra, Suiza, del 3 al 20 de febrero.

Consejo Interamericano Económico Social (CIES). 1963. Informe de la República Argentina para la Segunda Sesión de la Comisión Especial VI del Consejo Interamericano Económico Social, Buenos Aires. Kennedy, Robert F. 1965. Accomplishments under the Alliance, Alliance for Progress, 11/1965, Boston, Senate Papers, Box 9, Series Trips, 1964-1966, John F. Kennedy Library (JFKL).

Alianza para el Progreso. La Argentina y la Alianza para el Progreso. Obras en ejecución, Buenos Aires, s/f.

"Obituaries. John Heilman. USAID Official", en Washington Post, 27 de abril de 2002.

"Obituaries. Richard E. Lippincott", en Washington Post, 7 de agosto de 2003.

\section{Bibliografía}

Ardnt, Heinz. 1992. Desarrollo Económico. La historia de una idea, Buenos Aires, Rei argentina. de Pablo, Juan Carlos. 1979. La economía que yo hice, Buenos Aires, Ediciones El Cronista Comercial. 
Domenech, Enrique. 1971. The Alliance for Progress and the Inter-american Committee of the Alliance for Progress, Washington.

Fiszbein, Martín. 2011. "Instituciones e ideas en desarrollo. La planificación económica en la Argentina, 1945-1975", en Rougie, Marcelo (comp.). Estudios sobre la industria argentina, Buenos Aires, Lenguaje Claro.

Frigerio, Rogelio. 1969. "El carácter de la crisis crónica de la economía argentina y la estrategia para superarla", en Desarrollo y desarrollismo, Buenos Aires, Editorial Galerna.

García Bossio, Horacio. 2016. ¿Qué nos hace más Nación?. Desafíos del desarrollismo frondicistafrigerista, Buenos Aires, EDUNLA. 2o Edición (1ํo edición 2014).

Giglio, James y Rabe, Stephen. 2003. Debating the Kennedy Presidency, Lanham (Maryland), Rowman Littlefield Publishers.

Jáuregui, Aníbal. 2014. "La planificación en la Argentina del desarrollo (1955-1973)”, en Temas de historia argentina y americana, № 22, Buenos Aires, Universidad Católica Argentina, pp. 135-153.

Kvaternik, Eugenio. 2006. Polarización, perspectivas y casos: a la búsqueda de una tipología, Documento de Trabajo № 1, Año 1, Buenos Aires, Instituto de Ciencias Políticas y Relaciones Internacionales, Universidad Católica Argentina.

Montuschi, Luisa y Vázquez Presedo, Vicente. 1970. Plan y laissez-faire, en la economía contemporánea, Buenos Aires, Ediciones Macchi.

Núñez, Paula Gabriela, López, Silvana. 2014. "Lo rural y lo científico: La presencia de la Estación Experimental Regional Agropecuaria (EERA) INTA Bariloche 1962- 1968", en Estudios Rurales, N 7, Bernal, Centro de Estudios de la Argentina Rural, Universidad Nacional de Quilmes, Segundo Semestre.

Pereira, Enrique. 2017. “Carranza, Ing. Roque Guillermo”, en Diccionario Biográfico Nacional de la Unión Cívica Radical. Disponible en: http://diccionarioradical.blogspot.com.ar/.

Smulovitz, Catalina. 1988. Oposición y gobierno en los años de Frondizi, 1 y 2, Argentina, Centro Editor de América Latina (CEAL), Colección Biblioteca Política Argentina, № 213 y 214.

Stawski, Martin y Taroncher, Miguel. 2019. "La planificación y el CONADE: un análisis del Plan Nacional de Desarrollo, 1965-1969" en Odisio, Juan y Rougier, Marcelo (comps.), Estudios sobre planificación y desarrollo económico. Aportes para un diseño institucional estratégico, Buenos Aires, Lenguaje claro Editora.

Tearoti, Silvia Aurora. 2013. "El Plan de Desarrollo 1965-1969, un intento de planificación en la Argentina", en XIV Jornadas Interescuelas/Departamentos de Historia. Jornadas llevadas a cabo por el Departamento de Historia de la Facultad de Filosofía y Letras, Universidad Nacional de Cuyo, Mendoza.

Theberge, James D. 1964. La estructura internacional de la Alianza para el Progreso, Buenos Aires, Fundación de Investigaciones Básicas de la Realidad Americana.

Thomas, Leonar (ed.). 2006. Encyclopedia of the Developing World, Nueva York, Routledge.

Thomas, Leonard, et. alt. (eds). 2012. Encyclopedia of U.S-Latin American Relations, Vol. 1, Thousand Oaks (California), SAGE Publications.

Warren Nystrom, Jhon and Haverstock, Nathan. 1966. The Alliance for Progress, key to Latin America's development, Princeton, N.J., Van Nostrand. 